Check for updates

Cite this: Mater. Chem. Front., 2019, 3, 2617

Received 27th August 2019, Accepted 25th September 2019

DOI: 10.1039/c9qm00543a

rsc.li/frontiers-materials

\title{
Advances in the application of high pressure in carbon dots
}

\author{
Ting Geng, ${ }^{a}$ Cui Liu, ${ }^{b}$ Guanjun Xiao, (D) *a Siyu Lu (D) *c and Bo Zou (D) *a
}

\begin{abstract}
Carbon dots (CDs) have drawn significant attention due to their superior properties including outstanding water solubility, low toxicity, favorable biocompatibility, photobleaching resistance, high chemical stability, and ease of modification. The investigations on CDs are increasing steadily and distinctly focused. However, the use of high pressure in the study of CDs has been explored only in the past few years. These studies provide new insights into the nature of the structures and optical properties of CDs. Herein, we provided a review to introduce the recent advances in the application of high pressure in CDs to researchers from various areas and encourage them to dedicate themselves to this interesting exploration discipline.
\end{abstract}

\section{Introduction}

Carbon dots (CDs) are a new type of carbon-based zerodimensional materials with sizes less than $10 \mathrm{~nm} .{ }^{1}$ They have drawn significant attention due to their excellent advantages including favorable biocompatibility, low toxicity, outstanding water solubility, high sensitivity, tunable fluorescence emission and excitation, and high quantum yield. ${ }^{2,3}$ Due to these unexpected properties, CDs are promising in diverse research fields such as chemical and biological sensing, bioimaging, theranostics, light-emitting diodes, photocatalysts, and solar cells. ${ }^{1,2,4-7}$ During the past few years, research on the synthesis, properties, and applications of CDs has progressed rapidly, as reviewed by many independent groups. ${ }^{8-14}$

However, because of their numerous precursors and synthesis conditions, it is difficult to obtain a clear understanding of the relationship between the structures and optical properties of CDs. Thus, it is of significance to clarify their photoluminescence (PL) mechanisms. For CDs, many literature studies have reported that their PL is related to their sizes, zigzag sites, surface states and conjugated systems. ${ }^{9,15-21}$ Although great success has been achieved, the PL mechanism of CDs in relation to their special chemical structures is not very clear, and their applications need to be further explored. Compared to other physical and chemical modification technologies, ${ }^{22-24}$ high pressure techniques not only can significantly affect the electronic structures of materials

\footnotetext{
${ }^{a}$ State Key Laboratory of Superhard Materials, College of Physics, Jilin University Changchun, 130012,China.E-mail: xguanjun@jlu.edu.cn, zoubo@jlu.edu.cn ${ }^{b}$ Key Laboratory of Analytical Chemistry for Biology and Medicine (Ministry of Education), College of Chemistry and Molecular Sciences, and the Institute for Advanced Studies, Wuhan University, Wuhan 430072, China

${ }^{c}$ College of Chemistry and Molecular Engineering Zhengzhou University, 100 Kexue Road, Zhengzhou 450001, China. E-mail: sylu2013@zzu.edu.cn
}

without changing the chemical composition, but can also improve their optical properties. With the rapid development of high-pressure technology, especially the use of the diamond anvil cell (DAC) apparatus, studies in the high-pressure field are being conducted extensively. Exhilarating results of the studies on materials under high pressures have been obtained including enhanced structural stability, photoresponse enhancement, bandgap optimization, piezochromism, and metallization. ${ }^{25-34}$ Due to these excellent properties, these materials are promising in diverse research fields such as multifunctional semiconductor devices, mechanosensors, indicators of mechanohistory, security papers, optoelectronic devices, and data storage systems. ${ }^{35-38}$

In the past several years, high pressure, an environmentally friendly and efficient tool, has been used for the study of CDs. ${ }^{39-44}$ Great accomplishments have been made in piezochromic luminescence, capturing high pressure phases, and pressure-triggered aggregation-induced emission enhancement. This review summarizes the recent advances in the use of high pressure in CDs along with the relationships between the chemical structures and optical properties of these CDs. We hope to shed light on the current challenges and future potential of CDs for more multiple applications by briefly summarizing recent results to encourage researchers from various areas to dedicate themselves to this interesting exploration discipline.

\section{Piezochromic luminescence in CDs with the $\mathrm{sp}^{2}$ to $\mathrm{sp}^{3}$ transition.}

Luminescent materials are attracting considerable attention because of their inspiring, extensive application prospects in fluorescent switches and optical devices due to their sensitivity 
to environmental stimuli. ${ }^{45}$ Pressure is one of the most common natural external stimuli. Piezochromic materials show color changes attributed to mechanical grinding or external pressure, which are widely used in various fields. ${ }^{46}$

Yang and coworkers firstly reported new yellow emissive CDs (Y-CDs) as a new class of piezochromic materials. ${ }^{42}$ The Y-CDs were prepared by a solvothermal route from phthalandione and $o$-phenylenediamine with an average diameter of approximately $10 \mathrm{~nm}$. The Y-CDs showed obvious piezochromic luminescence under high pressure. They carried out all in situ high-pressure experiments using a symmetric diamond anvil cell apparatus (Fig. 1a). The fluorescence emission of the Y-CDs clearly showed a gradual blue-shift from yellow $(557 \mathrm{~nm})$ to blue-green (491 nm) as the applied pressure increased, which resulted from changes in the structures of carbon from $\mathrm{sp}^{2}$ to $\mathrm{sp}^{3}$ under external pressure (Fig. $1 \mathrm{~b}$ and $\mathrm{c}$ ). The pressures should be different to change chemical bonds and intermolecular interactions because the bond energy of covalent bonds is much larger that of hydrogen bonds. Therefore, very high pressure is required for the $\mathrm{sp}^{3}$ transition in carbon dots. The PL spectra of the Y-CDs were nearly identical as the applied pressure decreased from 22.84 to $0 \mathrm{GPa}$ (Fig. 1d). This is markedly different from traditional piezochromic materials. ${ }^{45}$ After two cycles of compression and decompression, the color change of Y-CDs under pressure was also irreversible. This fact was further manifested by the irreversible absorption spectrum (Fig. 1e). Moreover, they examined the electronic structures in the Y-CDs at ambient pressure and high pressure by first-principles

(a)
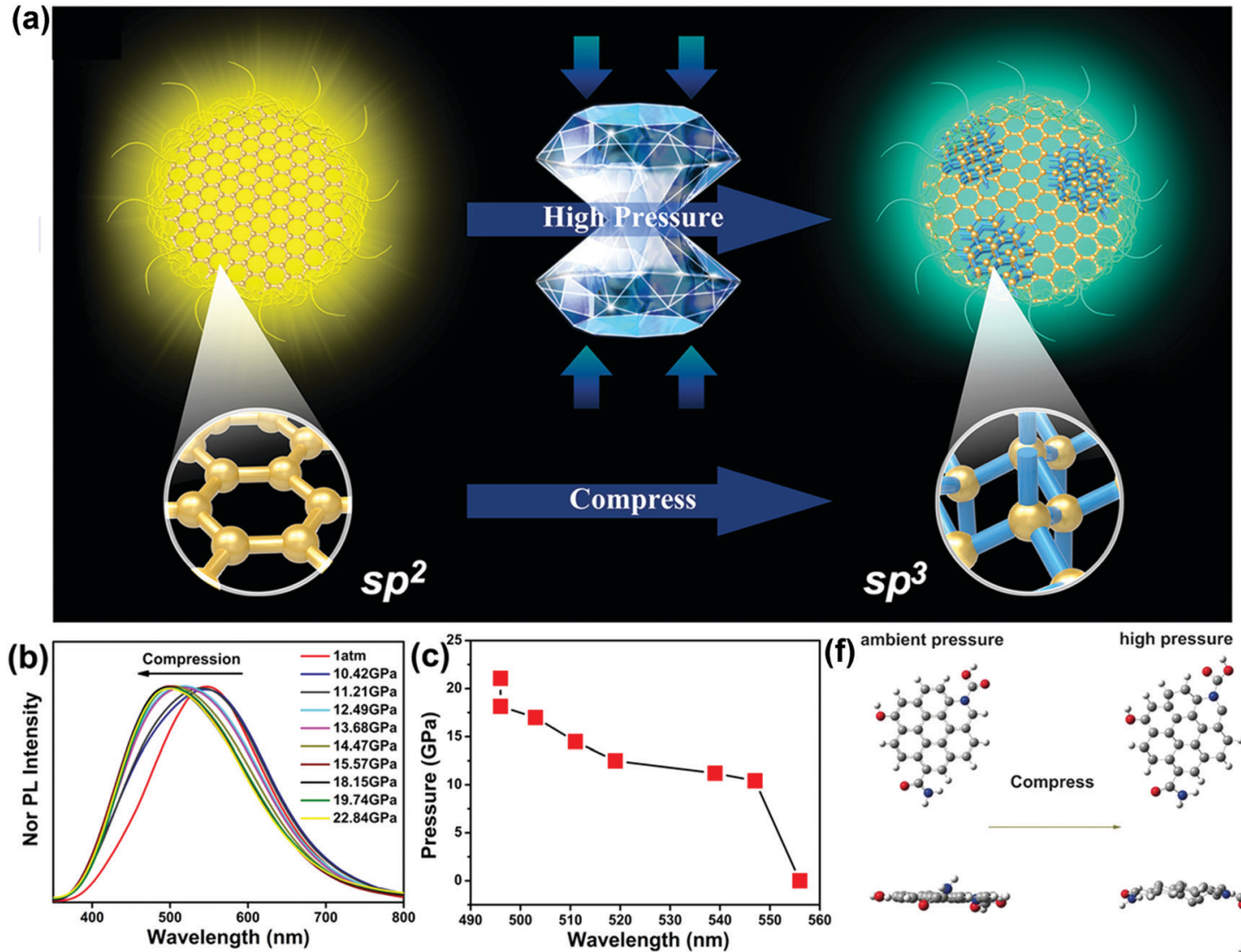

(f) ambient pressure

high pressure
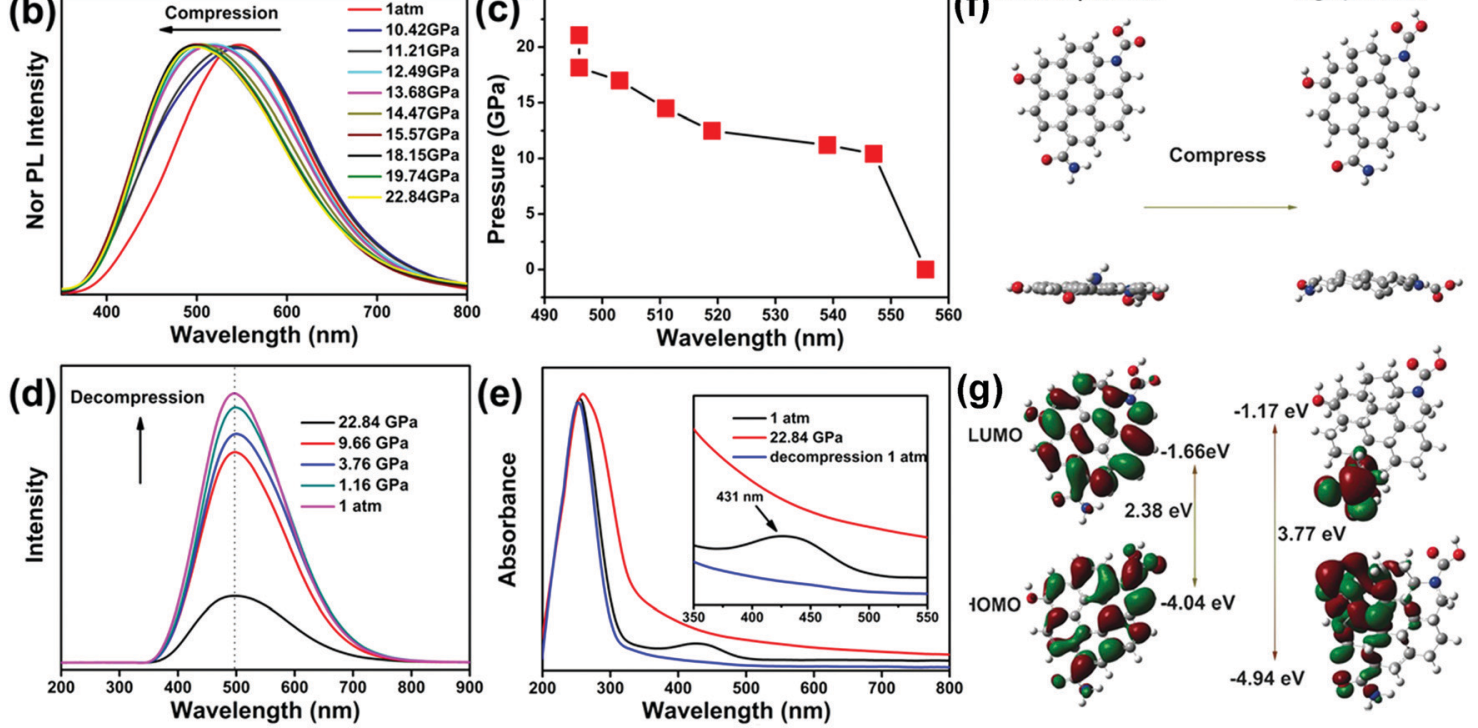

Fig. 1 (a) Schematic of the preparation and application of $Y$-CDs under high pressure. (b) PL spectra of $Y$-CDs with the increasing pressure. (c) PL location changes of $Y-C D$ s under external pressure. (d) PL spectra of $Y$-CDs upon decompression. (e) UV/Vis spectra of $Y$-CDs under selected pressures. Proposed fluorescence transition mechanism from $\mathrm{sp}^{2}$ to $\mathrm{sp}^{3}$ hybridization determined by first-principles calculations. (f) Top and side views of the structures under ambient and high pressures. (g) HOMO and LUMO orbitals of the ambient and high-pressure structures. Reproduced from ref. 42 with permission from Wiley. Copyright 2017. 
calculations (Fig. 1f and g). The computation further supported that the $\mathrm{sp}^{2}$ domains curved and transformed into $\mathrm{sp}^{3}$-hybridized domains under high pressure, leading to an ultimate color change from yellow to blue-green. This color-switchable feature of Y-CDs may have potential for applications in optical recording and pressure sensing materials. Meanwhile, the high pressure phase was successfully captured, which greatly increases the choice of materials available for a variety of applications.

\section{Pressure-controlled coupling of surface states and solvent effects on CDs.}

Due to their numerous precursors and synthetic conditions, it is difficult to clearly elucidate the relationships between the surface chemical structures and optical properties of CDs. It has been reported that the surface groups of CDs, such as pyridinic $\mathrm{N}$ and carbonyl O, play important roles in the PL of CDs. However, for carbon dots which contain only oxygen functional groups without chain compounds, chemical reactions are more likely to occur between the carbon dots and similar groups or solvent molecules under high pressure.

Recently, Pang et al. prepared C-dots with sizes of $1.7 \pm$ $0.3 \mathrm{~nm}$ by oxidation of carbon fiber powder. ${ }^{41}$ The PL emission wavelength of C-dots relies on the energy gap of the surface states, which is dictated by the coupling between carbonyl groups and the $\pi$-electron system. ${ }^{47,48}$ The PL of the C-dots exhibited a gradual blue-shift as the applied pressure increased from 0.07 to 0.53 GPa (Fig. 2a). The blue-shift PL of the C-dots under high pressure was attributed to the decrease of the carbonyl group content induced by pressure, which can enhance the addition reaction between the carbonyl groups and the nucleophilic structure of the C-dots. Upon further increase of the pressure, the emission wavelength of the C-dots no longer changed because the effects of $\pi-\pi$ stacking were weaker than the effects of the carbonyl groups. Generally speaking, the color changes of traditional mechanofluorochromic materials are completely or partially reversible. ${ }^{35}$ However, when the pressure was decreased from $1.54 \mathrm{GPa}$ to $1 \mathrm{~atm}$, the PL spectra of the C-dots were nearly identical (Fig. 2b); this indicates that the emission-related structures of the C-dots changed irrevocably during the pressure process. Meanwhile, C-dots- $\mathrm{NaOH}$ were obtained by treating C-dots in $\mathrm{NaOH}$ solution for $24 \mathrm{~h}$ at $200^{\circ} \mathrm{C}$. Hydrothermal treatment did not affect the physical size of C-dots-NaOH $(1.6 \pm 0.3 \mathrm{~nm})$. Compared with C-dots, the carbonyl and hydroxyl group contents of C-dots- $\mathrm{NaOH}$ decreased and the carboxyl group and $\mathrm{C}=\mathrm{C}$ contents increased. Therefore, the $\mathrm{PL}$ of C-dots-NaOH exhibited a blue-shift as the applied pressure was increased from 0 to $0.5 \mathrm{GPa}$, then exhibited a gradual redshift as the pressure increased (Fig. 2c). For further verification, the C-dots- $\mathrm{NaOH}$ were refluxed with hydriodic acid in acetic acid, in which the carbonyl and phenol groups can be reduced to hydroxy groups and aromatic hydrocarbons, respectively. The resulting sample was denoted as C-dots-NaOH-HI and had sizes of $1.6 \pm 0.3 \mathrm{~nm}$, indicating that the reduction exhibited no effects on the sizes of the C-dots. The PL spectra of C-dots-NaOH-HI exhibited a continuous red-shift with increasing pressure (Fig. 2d), indicating that the $\pi-\pi$ stacking induced by high pressure was the main reason for the red-shift of the PL. Upon complete release of pressure, the PL spectrum of C-dots-NaOH-HI recovered to its initial state. These results supported that the pressure-triggered red-shift of PL should be attributed to changes in the $\pi$-electron system under high pressure. In contrast, the decrease of carbonyl groups induces a blue-shift of the PL of the CDs (Fig. 2e).

The emission sites can be classed as carbonyl groupdominated or $\pi$-electron system-dominated. The two categories of emission sites can convert between each other though structure tuning with the aid of pressure. In a word, high pressure, as a clean and efficient tool, can achieve tunable mechanofluorochromicity by intentionally controlling the amount of carbonyl groups and the $\pi$-electron system.

Previous literature reports demonstrated that solvents can modulate the PL properties of CDs under atmospheric pressure. ${ }^{49-51}$ The study of the optical properties of CDs in different solvents under high pressure provides new insight into the relationships between the chemical structure and optical properties of CDs. Thus, it is of great scientific interest to tune the intrinsic emissions of CDs to better understand the origins of their luminescence and the influencing factors. High pressure, as a straightforward and efficient tool, can be used to study the structural and electronic properties of CDs; this can not only enable exploration of the changes in the CDs themselves under pressure, but can also enable exploration of the influence of different solvents on CDs under pressure.

$\mathrm{Qu}$ and coworkers reported solvent-dependent piezochromism of CDs using diamond anvil cells. ${ }^{39}$ The red-shift and blue-shift piezochromisms of CDs at high pressure were observed with $\mathrm{N}, \mathrm{N}$-dimethylformamide (DMF) and water as pressure transmitting mediums (PTMs), respectively. The color of the CDs in water changed from green to blue as the pressure increased from $0 \mathrm{GPa}$ to $25 \mathrm{GPa}$; meanwhile, green emission was retained in DMF even at $25 \mathrm{GPa}$ (Fig. $3 \mathrm{a}$ and b). The PL spectra of CDs in water firstly showed a gradual red-shift as the pressure increased from $0 \mathrm{GPa}$ to $1.8 \mathrm{GPa}$, which is attributed to the increasing $\pi-\pi$ stacking. Then, a blue-shift to $470 \mathrm{~nm}$ occurred upon further compression to $25 \mathrm{GPa}$. The PL of the CDs in water was partially recovered after high pressure relaxation (Fig. 3c). However, the PL of the CDs in DMF showed a continuous red-shift from 530 to $545 \mathrm{~nm}$ as the pressure increased from $0 \mathrm{GPa}$ to $25 \mathrm{GPa}$, which was attributed to enhanced $\pi-\pi$ stacking of the inner core under high pressure. Note that the PL profile of the CDs in DMF was totally recovered as the applied pressure decreased to $0 \mathrm{GPa}$, indicating that similar chemical reactions cannot occur between CDs and DMF molecules (Fig. 3d). Considering that water is a protic solvent, while DMF is an aprotic solvent, the reaction sites should be located on the electron withdrawing groups on the surface of the CDs, which can bond with hydroxyl groups in water molecules or chemically react with water molecules. For further 

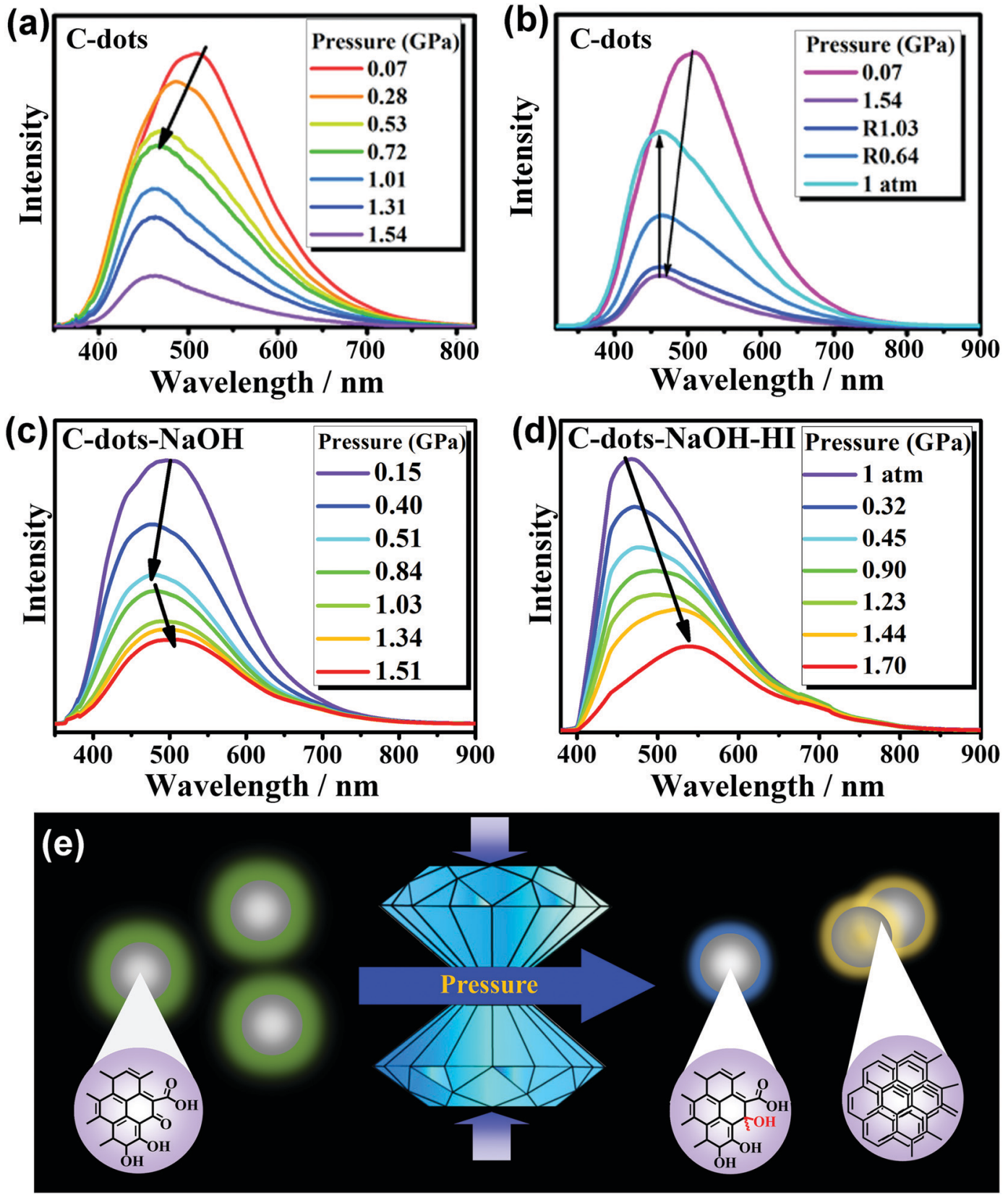

Fig. 2 (a) PL spectral changes of $C$-dots with the increasing pressure, (b) PL spectra of $C$-dots upon the decrease of external pressure to the ambient environment, pressure-dependent PL evolution of (c) $\mathrm{C}$-dots- $\mathrm{NaOH}$ and (d) $\mathrm{C}$-dots- $\mathrm{NaOH}-\mathrm{HI}$, and (e) an illustration of the PL and proposed structure changes of C-dots under high pressures. Reproduced from ref. 41 with permission from Wiley. Copyright 2018.

verification, they measured pressure-dependent PL spectra of the CDs in ethanol (Fig. 3e). The results showed that the PL spectra experienced a blue-shift from $530 \mathrm{~nm}$ to $440 \mathrm{~nm}$ with increasing pressure. The PL of the CDs in ethanol was partially recovered after high pressure relaxation (Fig. $3 \mathrm{f}$ ). These results further indicated that the blue-shifted piezochromism is related to protic solvents, such as water and ethanol.

In order to better understand the chemical reactions between water and CDs under high pressure, they carried out high-pressure experiments on CDs in water with high temperature treatment $\left(80{ }^{\circ} \mathrm{C}, 353 \mathrm{~K}\right)$ (Fig. $\left.3 \mathrm{~g}\right)$. The PL spectra after high pressure relaxation under high temperature showed unrecovered profiles and a more residual blue emissive band (Fig. 3h). Therefore, in summary, the red-shifted piezochromism was attributed to enhanced $\pi-\pi$ stacking, ${ }^{45}$ while the reversible and irreversible blue-shift piezochromism with water as the PTM was ascribed to pressure-induced enhanced intermolecular hydrogen bonding and addition reactions between water molecules and surface electron-withdrawing groups, respectively. This study examined the optical properties of CDs in different solvents using high pressure, which provides deeper insight into the fundamental origin of the luminescence of CDs and 

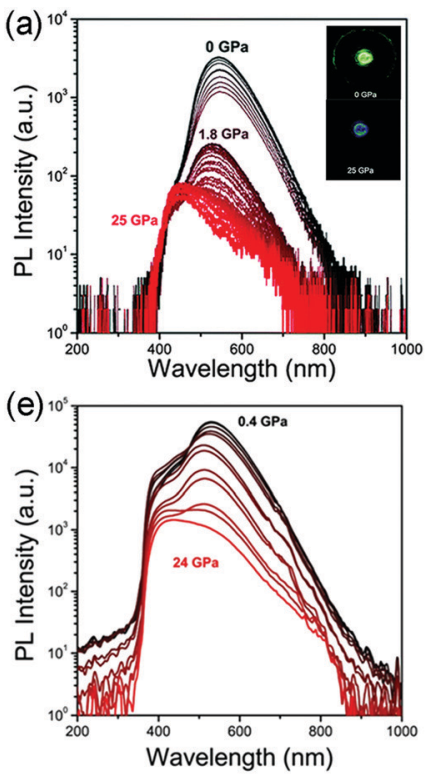
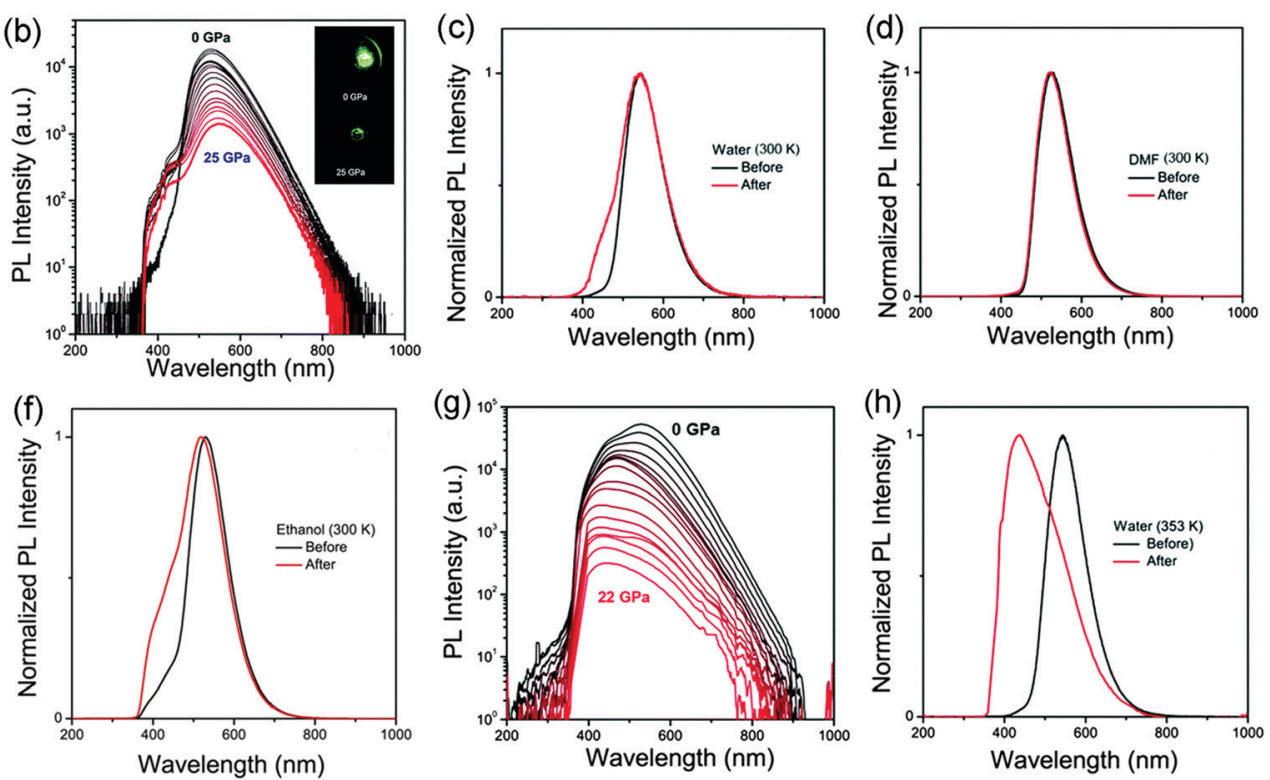

Fig. 3 PL spectral evolution and images of CDs in (a) water and (b) DMF with the increasing pressure from atmospheric pressure to $25 \mathrm{GPa}$. PL spectra before (black) and after (red) high pressure experiments with (c) water and (d) DMF as PTMs at room temperature (300 K). (e) PL spectral evolution and photographs of $\mathrm{CDs}$ in ethanol with increasing pressure from $0.4 \mathrm{GPa}$ to $24 \mathrm{GPa}$. (f) PL spectral evolution of CDs in ethanol under high pressure at room temperature $(300 \mathrm{~K})$. (g) PL spectral evolution of CDs in water at a high temperature of $353 \mathrm{~K}$. (h) PL spectra before (black) and after (red) high pressure experiments with water as the PTM at high temperature (353 K). Reproduced from ref. 39 with permission from The Royal Society of Chemistry.

into adjusting their intrinsic luminescence to promote their applications in different fields.

\section{Effects of high pressure on supramolecular interactions in CDs.}

Supramolecular interactions occur in most materials and play significant roles in their optical and physical properties. However, the study of regulation of the fluorescence of CDs by supramolecular interactions remains elusive. High pressure is particularly suitable to investigate these complex systems because the application of external pressure can lead to significant variations in hydrogen bonds and halogen bonds. For carbon dots whose surfaces contain polymer chains, supramolecular interactions between the polymer chains are dominant under high pressure. We recently reported on the supramolecular interactiontriggered fluorescence changes of $\mathrm{CDs}$ by investigating their piezochromic behaviors. ${ }^{40}$ The $\pi$-conjugated systems and hydroxy groups are critical for regulating the fluorescence of CDs under high pressure.

CDs-1 and CDs-2 with different hydrogen bond ratios were synthesized by adjusting the molar ratio of $p$-amino salicylic acid to citric acid. The PL peak of solid CDs-1 showed a gradual red-shift from 593 to $623 \mathrm{~nm}$ as the pressure increased from $1 \mathrm{~atm}$ to $17.8 \mathrm{GPa}$ (Fig. 4a). Meanwhile, the PL wavelength of CDs-2 exhibited a blue-shift from $526.5 \mathrm{~nm}$ to $510.0 \mathrm{~nm}$ as the pressure increased (Fig. 4b). The FT-IR spectra depict that CDs-2 contains more hydroxyl and carbonyl groups than CDs-1 (Fig. 4c). This indicates that compared with CDs-1, CDs-2 are formed with a greater ratio of hydrogen bonds. Considering the decreased $\pi-\pi$ stacking interactions due to the decrease in the benzene ring-containing precursor content, the hydroxyl and/or carbonyl-induced hydrogen bonds can cause fluorescence blueshifting under high pressure as the $\pi-\pi$ stacking interactions are weakened.

To validate the hydrogen-bond-caused blue-shifting effect and determine the specific groups inducing the blue-shift, we synthesized polyvinyl alcohol (PVA)/CDs-1 and PVA/CDs-2. These composites were fabricated using CPDs-1 solid powder and PVA powder with different mass ratios of $1: 1$ (15:15 mg) and 1:5 (15:75 mg). PVA/CDs-2 possessed more hydroxyl groups than CDs-1, as confirmed by FT-IR spectra. PVA/CDs-1 exhibited a red-shift as the pressure increased; this was also attributed to the $\pi-\pi$ stacking interactions, similar to that of the solid CDs-1. However, upon further increase in the applied pressure (from 11.3 to $20.1 \mathrm{GPa}$ ), the PL of PVA/CDs-1 exhibited a gradual blue-shift (Fig. 4d). The PL peak of solid PVA/CDs-2 under high pressure firstly showed a slight red-shift as the applied pressure increased from $1 \mathrm{~atm}$ to $4.3 \mathrm{GPa}$. Then, an obvious blue-shift occurred in the pressure range of 4.3 to 20.3 GPa (Fig. 4e). The pressure point at which PVA/CDs-2 shows a blue shift is lower than that of PVA/CDs-1.

Accordingly, increasing the number of hydrogen-bonds by incorporating more hydroxyl-rich PVA in CDs-1 induced an obvious PL blue-shift by hydrogen-bonding effects as a result of the extremely lowered $\pi-\pi$ stacking interaction-induced redshifting effect. In addition, high-pressure IR measurements showed a shift towards low wavenumbers of the $\mathrm{O}-\mathrm{H}$ stretching frequency up to $17.9 \mathrm{GPa}$, and the vibrational mode was gradually broadened beyond that pressure (Fig. 4f). This behavior is characteristic of the formation of hydrogen bonds. ${ }^{52}$ 

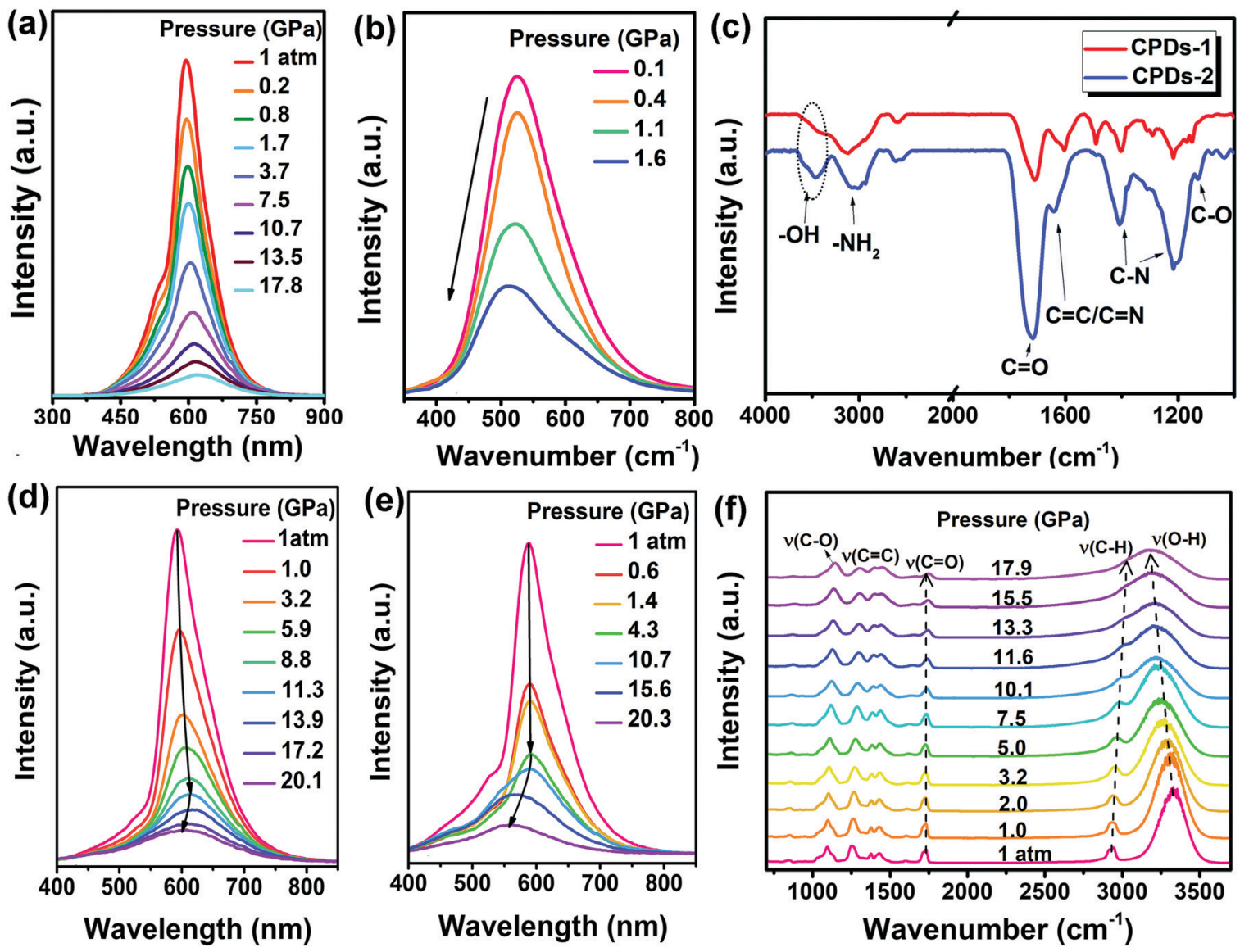

Fig. 4 (a) PL spectral changes of solid CDs-1 under high pressure, (b) PL spectral changes of CDs-2 against pressure (the black arrows clearly show the evolution of the PL spectra with increasing pressure), (c) FT-IR spectra of CDs-1 and CDs-2, (d) PL spectral changes of PVA/CDs-1 under high pressure, (e) PL spectral changes of PVA/CDs-2 as the applied pressure is gradually increased. (f) High-pressure IR spectra of PVA/CDs-2 at room temperature. Reproduced from ref. 40 with permission from The Royal Society of Chemistry.

The $\mathrm{C}=\mathrm{O}$ stretching vibration $\nu(\mathrm{C}=\mathrm{O})$ absorption intensity and frequency changed slightly as the pressure increased, which implies that the carbonyl group has almost no effect on the blue-shifting of PL under high pressure. High-pressure IR experiments provided further in-depth insights into the hydrogen bond-induced blue-shift of PL.

Likewise, Wu and coworkers proposed a facile and one-step solvothermal approach to fabricate nitrogen-doped carbon dots (N-CDs). ${ }^{43}$ The absolute photoluminescence quantum yield (PLQY) reached 56.1\%, which is far higher than that of the pristine CDs (26.9\%) (Fig. 5a). As the applied pressure was increased from 0.07 to $0.88 \mathrm{GPa}$, the PL spectrum of the N-CDs initially displayed a gradual red-shift from 516 to $558 \mathrm{~nm}$, which is attributed to the enhanced $\pi-\pi$ stacking interactions (Fig. 5b). As the pressure further increased to $5.18 \mathrm{GPa}$, the PL spectra exhibited a gradual blue-shift from 558 to $520 \mathrm{~nm}$, resulting from the hydrogen bond effect. Accordingly, the color of the N-CDs gradually darkened as the applied pressure increased (Fig. 5d). The PL spectra of the as-synthesized N-CDs firstly showed a gradual red-shift and then a blue-shift as the pressure decreased to ambient conditions (Fig. 5c), indicating the partial reversibility of the piezochromic luminescence of N-CDs. Benefiting from their outstanding water solubility, low toxicity, and high stability, the piezochromic N-CDs may be promising candidates as piezochromic materials for the sensitive detection of external pressure stimuli.

\section{Pressure-triggered aggregation- induced emission enhancement in CDs}

High pressure can be a robust tool to enhance the photoluminescence efficiency of materials and provide insights into their structures and optical properties. Although some great achievements in pressure-induced emission and enhancement have been gained, ${ }^{25,53-55}$ fewer literature reports are related to the emission enhancement of CDs under external pressure. Recently, Lu and coworkers obtained pressure-triggered aggregationinduced emission enhancement in CDs with red emission (R-CDs) synthesized using 1,3,5-benzenetrithiol as the raw material. ${ }^{44}$ In situ high-pressure experiments were carried out using a symmetric diamond anvil cell apparatus at room temperature (Fig. 6a). The R-CDs were composed of aromatic rings crosslinked by weak interactions. The aromatic rings in the R-CDs underwent dynamic intraparticle oscillations against each other, resulting in consumption of the excited state energy by 
(a)
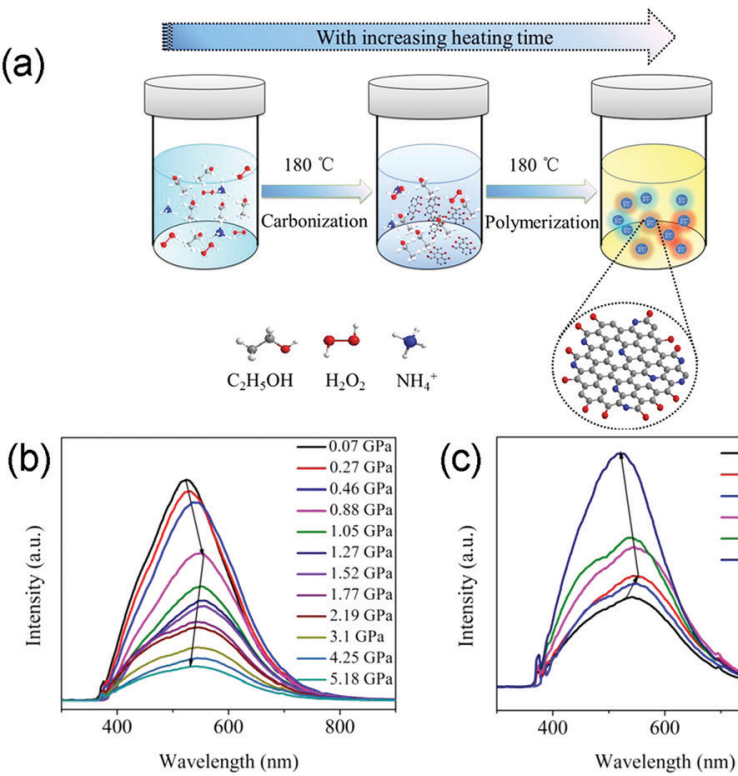

(c)

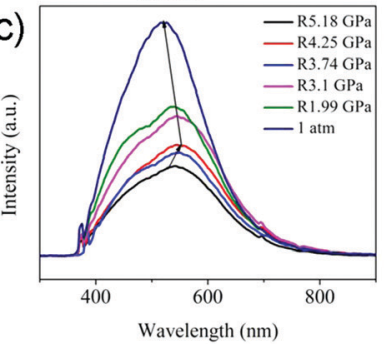

(d)

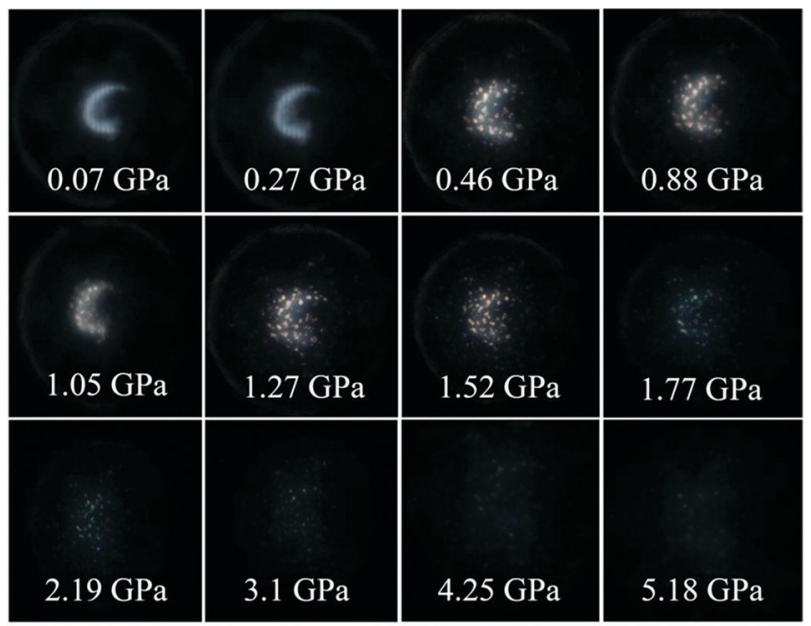

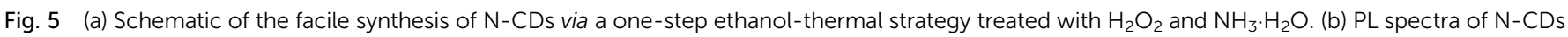

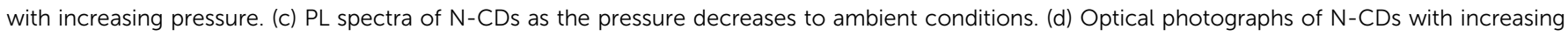
pressure. Reproduced with permission from ref. 43. Copyright 2018 American Chemical Society.

nonradiative decay. Upon compression, the intraparticle oscillations were greatly restricted due to physical constraints. This restriction blocks the non-radiative pathways and opens up the radiative channels. Therefore, the R-CDs exhibited distinct emission enhancement at the starting pressure of $0.05 \mathrm{GPa}$, and the fluorescence intensity within the applied pressure range of 0.05 to $0.5 \mathrm{GPa}$ was superior to that at 1 atm (Fig. 6b). This behavior was due to pressure-triggered aggregation-induced emission. The prepared R-CDs can be effectively exploited as promising pressure-sensing materials due to the low applied pressure (tens of $\mathrm{MPa}$ ) for their fluorescence enhancement. When the pressure was increased above 1.2 GPa, the intermolecular distance of the R-CDs continued to shrink, leading to agglomeration of the R-CDs and thus to a decrease in fluorescence intensity. The normalized fluorescence intensity gradually increased and then experienced a decrease (Fig. 6c). During the process of decompression, the fluorescence intensity gradually increased and recovered its level at atmospheric pressure (Fig. 6e and f). UV-vis spectroscopy also demonstrated this reversible phenomenon (Fig. 6d). The pressure only influences the state of aggregation of the R-CDs without destroying their structures (Fig. 6g). The reversible process after the cycle of compression and decompression was thus expected. Time-dependent density functional theory calculations further supported that the R-CDs were brought close together, accompanied by suppressed intraparticle oscillations, under mild pressure. The compressed R-CDs possessed strong intramolecular charge transfer from their natural transition orbitals, which is responsible for the enhanced emission under high pressure. This research provides new ideas for the development of novel piezochromic materials in the future.

\section{Summary and outlook}

Studies on CDs have been expanding rapidly due to their propitious applications in various fields. Here, we focused on the latest progress and future perspectives in the exciting research on high pressure advances in CDs, including welldesigned structures to obtain specific properties. Pressure processing is being used as a robust strategy to explore the fluorescence mechanisms of CDs. Pressure, as a thermodynamic variable independent of temperature and composition, can effectively change the atomic arrangements, electronic structures and interactions between molecules of a material. The pressure effects are different for materials with different structures in the same class. Therefore, the pressure response to carbon dots with different structures is also different. In this review, we summarize the recent reports on six kinds of piezochromic luminescence in carbon dots and the underlying mechanisms. In the process of applying pressure, both intermolecular interactions and intramolecular interactions occur simultaneously. These two interactions can lead to distortion and even fracture of chemical bonds, crosslinking between polymer chains on the surface of carbon dots, or effects such as formation of hydrogen bonds, formation or dissociation of electrostatic interactions between surface groups and $\pi-\pi$ stacking between carbon dots. The red-shifted piezochromism was attributed to enhanced $\pi-\pi$ stacking, the reversible blueshifted piezochromism was ascribed to pressure-enhanced intermolecular hydrogen bonding, and the irreversible blueshifted piezochromism was attributed to hybrid state transitions or addition reactions between specific surface groups and solvent molecules. The emission enhancement originated from pressure-triggered aggregation by suppressing intraparticle oscillations. Increasing efforts are required to improve the 

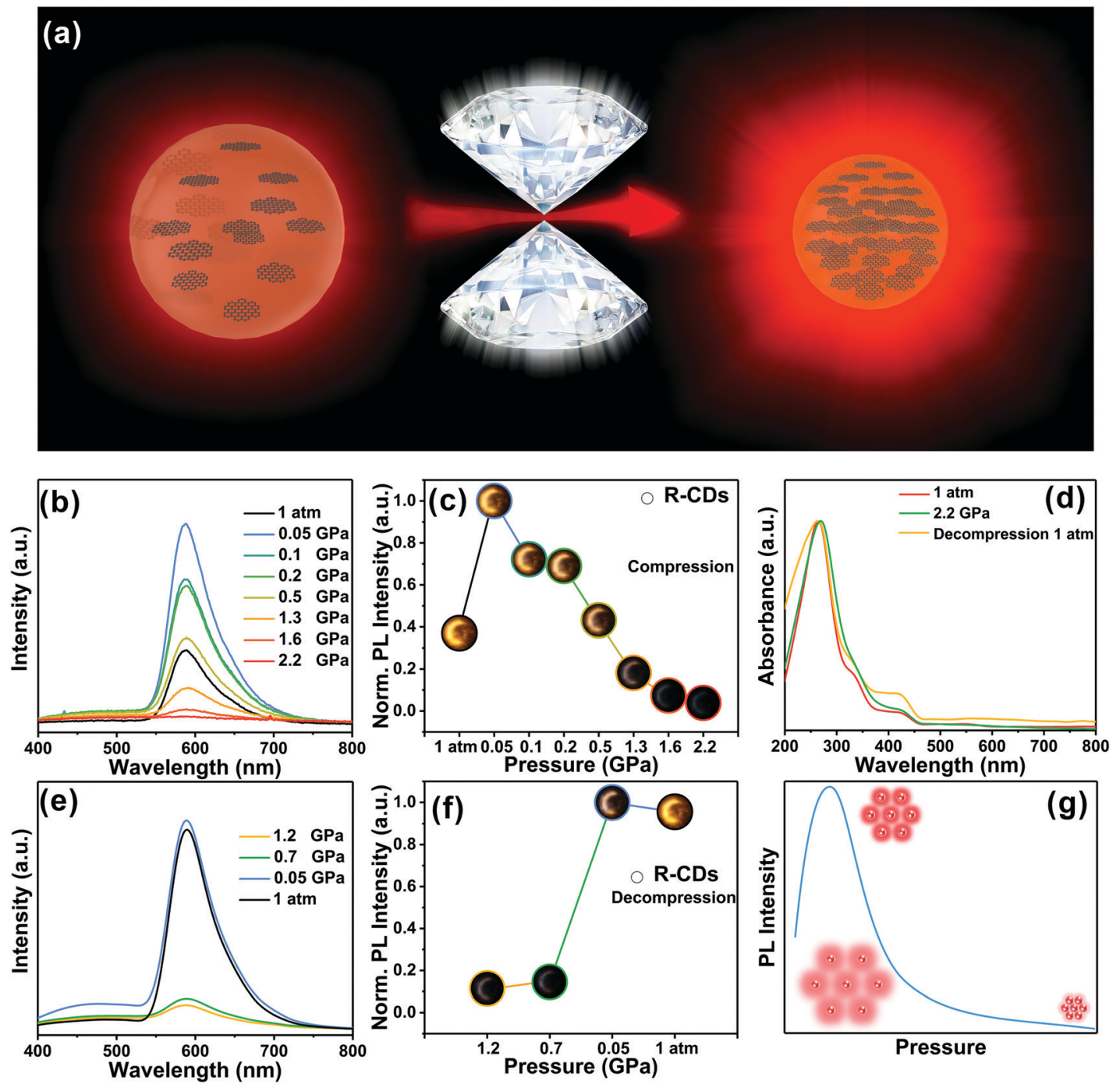

Fig. 6 (a) Illustration of the fluorescence enhancement of R-CDs under high pressure; (b and e) PL spectra of the R-CDs as the pressure increases and then decreases to ambient conditions. (c and f) PL emissive peak changes of the R-CDs with increasing and decreasing pressure. (d) UV-Vis spectra of the $\mathrm{R}-\mathrm{CDs}$ as the pressure increases and decreases to ambient conditions. (g) Reversible process of the R-CDs. Reproduced from ref. 44 with permission from The Royal Society of Chemistry.

luminescence performance of these materials for highperformance applications. MXene $\mathrm{Ti}_{3} \mathrm{C}_{2}$ quantum dots exhibited high stability under high pressure, simply changing from cool white emission to warm white emission. ${ }^{56}$ Therefore, high pressure can be used not only to investigate the related PL mechanisms and explore PL color regulation but also to achieve high stability.

Research into the modification and exploration of various properties using high pressure to obtain better performance of CDs has made remarkable progress. However, the structureproperty relationships and underlying mechanisms of the special chemical structures of CDs are not very clear, and their emerging applications need to be further explored. Tremendous work remains to be performed to overcome a range of current scientific issues. As is known, the PL of CDs is affected by many factors, such as their sizes, surface states and conjugate systems. Therefore, uncertain formation processes and complicated synthetic conditions represent great challenges for the study of CDs. In addition, pressure generally quenches fluorescence. High PLQY and intense brightness are vital for the wide application of CDs.

It appears that, along with development, if the current scientific issues are well resolved by scientists and engineers, the study of the effects of high pressure on CDs will provide a promising platform for future development and commercialization of multifunctional applications. Meanwhile, CDs will be used commercially in various areas and compete with traditional semiconductor quantum dots.

\section{Conflicts of interest}

There are no conflicts to declare. 


\section{Acknowledgements}

This work is supported by the National Science Foundation of China (No. 21725304, 11774125, 21673100 and 21805021), Chang Jiang Scholars Program of China (No. T2016051), Changbai Mountain Scholars Program (No. 2013007), National Defense Science and Technology Key Laboratory Fund (No. 6142A0306010917), Jilin Provincial Science \& Technology Development Program (No. 20190103044JH), Scientific Research Planning Project of the Education Department of Jilin Province (No. JJKH20180118KJ), and China Postdoctoral Science Foundation Funded Project (2018M631057).

\section{Notes and references}

1 F. Yuan, S. Li, Z. Fan, X. Meng, L. Fan and S. Yang, Nano Today, 2016, 11, 565-586.

2 M. Sun, C. Liang, Z. Tian, E. V. Ushakova, D. Li, G. Xing, S. Qu and A. L. Rogach, J. Phys. Chem. Lett., 2019, 10, 3094-3100.

3 S. N. Baker and G. A. Baker, Angew. Chem., Int. Ed., 2010, 49, 6726-6744.

4 D. Li, P. Jing, L. Sun, Y. An, X. Shan, X. Lu, D. Zhou, D. Han, D. Shen, Y. Zhai, S. Qu, R. Zbořil and A. L. Rogach, Adv. Mater., 2018, 30, 1705913.

$5 \mathrm{X} . \mathrm{Wu}, \mathrm{J}$. Zhao, L. Wang, M. Han, M. Zhang, H. Wang, H. Huang, Y. Liu and Z. Kang, Appl. Catal., B, 2017, 206, 501-509.

6 X. Bao, Y. Yuan, J. Chen, B. Zhang, D. Li, D. Zhou, P. Jing, G. Xu, Y. Wang, K. Holá, D. Shen, C. Wu, L. Song, C. Liu, R. Zbořil and S. Qu, Light: Sci. Appl., 2018, 7, 91.

7 F. Yuan, Z. Wang, X. Li, Y. Li, Z. A. Tan, L. Fan and S. Yang, Adv. Mater., 2017, 29, 1604436.

8 R. Das, R. Bandyopadhyay and P. Pramanik, Mater. Today Chem., 2018, 8, 96-109.

9 F. Yan, Y. Jiang, X. Sun, Z. Bai, Y. Zhang and X. Zhou, Microchim. Acta, 2018, 185, 424.

10 M. Kaur, M. Kaur and V. K. Sharma, Adv. Colloid Interface Sci., 2018, 259, 44-64.

11 D. L. Zhao and T.-S. Chung, Water Res., 2018, 147, 43-49.

12 M. J. Molaei, Talanta, 2019, 196, 456-478.

13 W. Meng, X. Bai, B. Wang, Z. Liu, S. Lu and B. Yang, Energy Environ. Mater., 2019, 2, 172-192.

14 T. Feng, S. Zhu, Q. Zeng, S. Lu, S. Tao, J. Liu and B. Yang, ACS Appl. Mater. Interfaces, 2018, 10, 12262-12277.

15 H. Li, X. He, Z. Kang, H. Huang, Y. Liu, J. Liu, S. Lian, C. H. A. Tsang, X. Yang and S.-T. Lee, Angew. Chem., Int. Ed., 2010, 49, 4430-4434.

16 X. T. Zheng, A. Ananthanarayanan, K. Q. Luo and P. Chen, Small, 2015, 11, 1620-1636.

17 Y.-P. Sun, B. Zhou, Y. Lin, W. Wang, K. A. S. Fernando, P. Pathak, M. J. Meziani, B. A. Harruff, X. Wang, H. Wang, P. G. Luo, H. Yang, M. E. Kose, B. Chen, L. M. Veca and S.-Y. Xie, J. Am. Chem. Soc., 2006, 128, 7756-7757.

18 Q. Lou, S. Qu, P. Jing, W. Ji, D. Li, J. Cao, H. Zhang, L. Liu, J. Zhao and D. Shen, Adv. Mater., 2015, 27, 1389-1394.
19 B. van Dam, H. Nie, B. Ju, E. Marino, J. M. J. Paulusse, P. Schall, M. Li and K. Dohnalová, Small, 2017, 13, 1702098.

20 C. Liu, L. Bao, M. Yang, S. Zhang, M. Zhou, B. Tang, B. Wang, Y. Liu, Z.-L. Zhang, B. Zhang and D.-W. Pang, J. Phys. Chem. Lett., 2019, 10, 3621-3629.

21 Q.-L. Zhao, Z.-L. Zhang, B.-H. Huang, J. Peng, M. Zhang and D.-W. Pang, Chem. Commun., 2008, 5116-5118.

22 D. Amgar, A. Stern, D. Rotem, D. Porath and L. Etgar, Nano Lett. , 2017, 17, 1007-1013.

23 F.-S. Zu, P. Amsalem, I. Salzmann, R.-B. Wang, M. Ralaiarisoa, S. Kowarik, S. Duhm and N. Koch, Adv. Opt. Mater., 2017, 5, 1700139.

24 Q. A. Akkerman, V. D’Innocenzo, S. Accornero, A. Scarpellini, A. Petrozza, M. Prato and L. Manna, J. Am. Chem. Soc., 2015, 137, 10276-10281.

25 Y. Shi, Z. Ma, D. Zhao, Y. Chen, Y. Cao, K. Wang, G. Xiao and B. Zou, J. Am. Chem. Soc., 2019, 141, 6504-6508.

26 Z. Ma, F. Li, G. Qi, L. Wang, C. Liu, K. Wang, G. Xiao and B. Zou, Nanoscale, 2019, 11, 820-825.

27 Q. Zeng, K. Wang and B. Zou, J. Am. Chem. Soc., 2017, 139, 15648-15651.

28 Z. Wang, S. Guo, H. Li, B. Wang, Y. Sun, Z. Xu, X. Chen, K. Wu, X. Zhang, F. Xing, L. Li and W. Hu, Adv. Mater., 2019, 31, 1805630.

29 H. Zhu, T. Cai, M. Que, J.-P. Song, B. M. Rubenstein, Z. Wang and O. Chen, J. Phys. Chem. Lett., 2018, 9, 4199-4205.

30 G. Xiao, Y. Cao, G. Qi, L. Wang, C. Liu, Z. Ma, X. Yang, Y. Sui, W. Zheng and B. Zou, J. Am. Chem. Soc., 2017, 139, 10087-10094.

31 L. Zhang, C. Liu, L. Wang, C. Liu, K. Wang and B. Zou, Angew. Chem., Int. Ed., 2018, 57, 11213-11217.

32 H. Wu, Z. Wang and H. Fan, J. Am. Chem. Soc., 2014, 136, 7634-7636.

33 G. Xiao, X. Yang, X. Zhang, K. Wang, X. Huang, Z. Ding, Y. Ma, G. Zou and B. Zou, J. Am. Chem. Soc., 2015, 137, 10297-10303.

34 H. Zhu, T. Cai, M. Que, J.-P. Song, B. M. Rubenstein, Z. Wang and O. Chen, J. Phys. Chem. Lett., 2018, 9, 4199-4205.

35 Y. Wang, X. Lü, W. Yang, T. Wen, L. Yang, X. Ren, L. Wang, Z. Lin and Y. Zhao, J. Am. Chem. Soc., 2015, 137, 11144-11149.

36 L. Wang, K. Wang, G. Xiao, Q. Zeng and B. Zou, J. Phys. Chem. Lett., 2016, 7, 5273-5279.

37 R. Fu, Y. Chen, X. Yong, Z. Ma, L. Wang, P. Lv, S. Lu, G. Xiao and B. Zou, Nanoscale, 2019, 11, 17004-17009.

38 Y. Sagara, T. Mutai, I. Yoshikawa and K. Araki, J. Am. Chem. Soc., 2007, 129, 1520-1521.

39 P. Jing, D. Han, D. Li, D. Zhou, D. Shen, G. Xiao, B. Zou and S. Qu, Nanoscale Horiz., 2019, 4, 175.

40 T. Geng, T. Feng, Z. Ma, Y. Cao, Y. Chen, S. Tao, G. Xiao, S. Lu, B. Yang and B. Zou, Nanoscale, 2019, 11, 5072-5079.

41 C. Liu, G. Xiao, M. Yang, B. Zou, Z.-L. Zhang and D.-W. Pang, Angew. Chem., Int. Ed., 2018, 57, 1893-1897.

42 S. Lu, G. Xiao, L. Sui, T. Feng, X. Yong, S. Zhu, B. Li, Z. Liu, B. Zou, M. Jin, J. S. Tse., H. Yan and B. Yang, Angew. Chem., Int. Ed., 2017, 56, 6187-6191. 
43 Y. Zhan, T. Geng, Y. Liu, C. Hu, X. Zhang, B. Lei, J. Zhuang, X. Wu, D. Huang, G. Xiao and B. Zou, ACS Appl. Mater. Interfaces, 2018, 10, 27920-27927.

44 Q. Wang, S. Zhang, B. Wang, X. Yang, B. Zou, B. Yang and S. Lu, Nanoscale Horiz., 2019, 4, 1227-1231.

45 Y. Dong, B. Xu, J. Zhang, X. Tan, L. Wang, J. Chen, H. Lv, S. Wen, B. Li, L. Ye, B. Zou and W. Tian, Angew. Chem., Int. Ed., 2012, 51, 10782-10785.

46 Y. Cao, G. Qi, C. Liu, L. Wang, Z. Ma, K. Wang, F. Du, G. Xiao and B. Zou, J. Phys. Chem. C, 2018, 122, 9332-9338.

47 L. Bao, Z.-L. Zhang, Z.-Q. Tian, L. Zhang, C. Liu, Y. Lin, B. Qi and D.-W. Pang, Adv. Mater., 2011, 23, 5801-5806.

48 B.-P. Qi, H. Hu, L. Bao, Z.-L. Zhang, B. Tang, Y. Peng, B.-S. Wang and D.-W. Pang, Nanoscale, 2015, 7, 5969-5973.

49 H. Wang, C. Sun, X. Chen, Y. Zhang, V. L. Colvin, Q. Rice, J. Seo, S. Feng, S. Wang and W. W. Yu, Nanoscale, 2017, 9, 1909-1915.
50 T. Zhang, J. Zhu, Y. Zhai, H. Wang, X. Bai, B. Dong, H. Wang and H. Song, Nanoscale, 2017, 9, 13042-13051.

51 A. Sciortino, E. Marino, B. V. Dam, P. Schall, M. Cannas and F. Messina, J. Phys. Chem. Lett., 2016, 7, 3419-3423.

52 S. A. Medvedev, M. I. Eremets, J. Evers, T. M. Klapötke, T. Palasyuk and I. A. Trojan, Chem. Phys., 2011, 386, 41-44. 53 G. Xiao, Y. Wang, D. Han, K. Li, X. Feng, P. Lv, K. Wang, L. Liu, S. A. T. Redfern and B. Zou, J. Am. Chem. Soc., 2018, 140, 13970-13975.

54 Z. Ma, Z. Liu, S. Lu, L. Wang, X. Feng, D. Yang, K. Wang, G. Xiao, L. Zhang, S. A. T. Redfern and B. Zou, Nat. Commun., 2018, 9, 4506.

55 Y. Gu, K. Wang, Y. Dai, G. Xiao, Y. Ma, Y. Qiao and B. Zou, J. Phys. Chem. Lett., 2017, 8, 4191-4196.

56 S. Lu, L. Sui, Y. Liu, X. Yong, G. Xiao, K. Yuan, Z. Liu, B. Liu, B. Zou and B. Yang, Adv. Sci., 2019, 6, 1801470. 